Istituto di Genetica Medica e Gemellologia «G. Mendel»(Roma)

Direttore: Prof. L. Gedda

\title{
Considerazioni su una famiglia di granulopenici
}

\section{Giuseppina Torrioli Riggio}

La granulopenia ciclica familiare non ha fatto la sua comparsa sull'orizzonte della patologia come una forma subito definita ed a sè stante. Essa è stata sospettata infatti da vari autori che in alcuni stati granulopenici cronici, di aspetto clinico diversissimo, hanno notato ondulazioni cicliche della granulopenia, cui corrispondeva un più o meno accentuato variare periodico del quadro clinico corrispondente. Basti ricordare la notissima sindrome di Felty, interpretata quasi universalmente come un'artrite settica, splenomegalica, con neutropenia da probabile ipersplenismo secondario. In alcuni casi, come quello di Löffler e Maier e come quello di Müller e Meyrieux (I948) presentarono un chiaro andamento ciclico della neutropenia con un periodo di due tre settimane, Quasi contemporaneamente si andavano raccogliendo altri casi in cui la sindrome di Felty era totalmente assente ed in cui invece il quadro clinico era dominato da una mucosite prevalentemente buccale a tipo settico. Così i casi di Imerslung ( 1942 ), Vahlquist (1946), fino ai 16 casi raccolti da Reimann e de Berardinis e a quelli di Sturgis riportati nel suo trattato del I955. Quest'ultimo autore accetta la denominazione di «Gengivostomatite acuta ricorrente infettiva con neutropenia ".

Contemporaneamente si andava studiando il comportamento delle agranulocitosi croniche ed anche in queste alcuni casi mostrarono oscillazioni più o meno regolari nella neutropenia che portò a sospettare la primitività di quest'ultima. Altri autori infine notarono la familiarità della forma.

Data questa molteplicità di origine del concetto di neutropenia ciclica familiare non meraviglia il fatto che anche oggi esista una profonda 'diversità di vedute sul meccanismo patogenetico della malattia.

Gli AA. che sono partiti dalla ciclicità delle manifestazioni cliniche a carattere settico si sono piuttosto orientati verso una variazione dell'agente etiologico batterico o virale cui corrispondesse una varia risposta organica. Sturgis per la gengivostomatite non esclude la possibilità di un virus a ricorrenti attacchi. Anche per la ciclizzazione nella sindrome di Felty l'ipotesi di un'attività periodica dello sconosciuto agente infettivo ha trovato sostenitori.

Gli AA. invece che come Reimann e de Berardinis hanno studiato più casi a manifestazioni cliniche differenti, si sono indirizzati più verso una variazione pri- 
mitivamente ematologica cui conseguivano oscillazioni del quadro settico. Orientati in questo senso si sono naturalmente posti la domanda sul meccanismo patogenetico

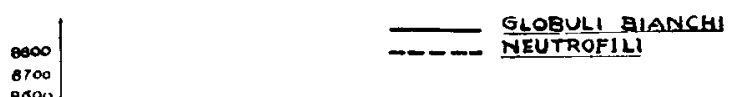

8000

8400

8300

e 200

$0+00$
0000

7900

7800

7500

7400
7300

7300
7200

7100

6900

6900
6800
6700

6700
0000

0000

0400

0300

0200
6100

6000
5900

5900
5800

5700

5000

5400

5300

5200
5100

5000

4900

4800

4700

4600

4500
4400

4300

4200

4100

4000

asoo

3700

3000

3900

$\$ 00$

3300
3200

3200

3000

2900

2000

2700

2000 G10001

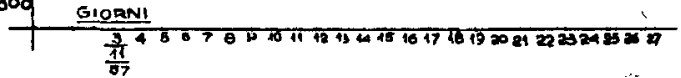

Grafico I - Francesco (candidato)

Il presente grafico, come $\mathrm{i}$ successivi, mette in evidenza l'andamento ciclico dei granulociti neutrofili e quello corrispondente del numero dei globuli bianchi. determinante la granulopenia ciclica:

Stabilire cioè in quale organo o sistema la ciclicità primitivamente risiedesse. Tre sono le fondamentali possibilità da mettere in discussione: a) attività midollare in sè; $b$ ) milza come organo mielodepressore e mieloregolatore; $c$ ) centri"regolatori neurormonici.

Gli AA. infine che colpirono fin dall'inizio la familiarità della forma si orientarono naturalmente verso una origine genotipica. Ammettere questo non significa però escludere l'importanza delle tre ipotesi patogenetiche su esposte intese come modi di manifestazione nel fenotipo del carattere genico. Riteniamo anzi che oggi il problema fondamentale sia precisamente questo, sia per il suo valore dottrinale, sia per la sua importanza nella impostazione di una corretta terapia degli ammalati. Torneremo quindi su questo punto dopo aver riportato $\mathrm{i}$ dati da noi raccolti.

Venne per primo alla nostra osservazione un ragazzo, Francesco, di i 8 anni inviato a visita specialistica dal curante. Nell'anamnesi patologica risultavano: all'età di 2 mesi enterocolite prolungata e recidivante. Morbillo nella seconda infanzia. Parotite a io anni; a I 3 dermatosi alla piega dell'orecchio e alle pliche ascellari e inguinali, riportabili ad una infezione da microsporon. Fistola coccigea operata a 8 anni. Dai 16 anni furuncoli a decorso lento e recidivante. L'esame obbiettivo generale era negativo per quel che riguarda gli apparati respiratorio, cardiovascolare e gli organi addominali. Anche la milza era nei limiti 
normali. Sulla cute delle gambe presentava nodi infiltrati, duri, di aspetto verrucoso, ulcerati. In altre parti del corpo erano visibili cicatrici violacee da pregressi furuncoli. La pressione era $\frac{\mathrm{I} 25}{80}$. La reazione di Wasserman negativa. Prima delle

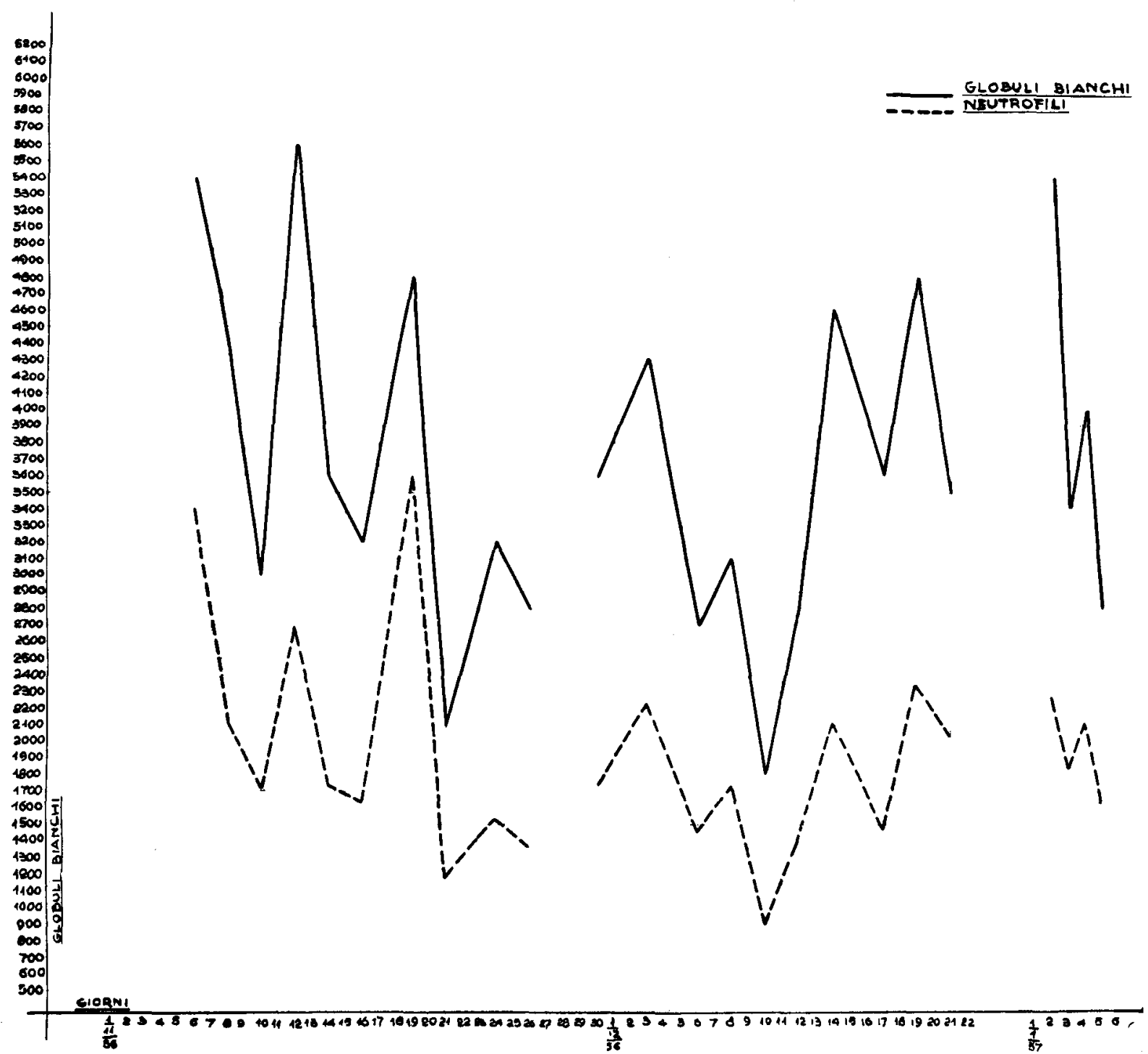

Grafico 2 - Ugo 


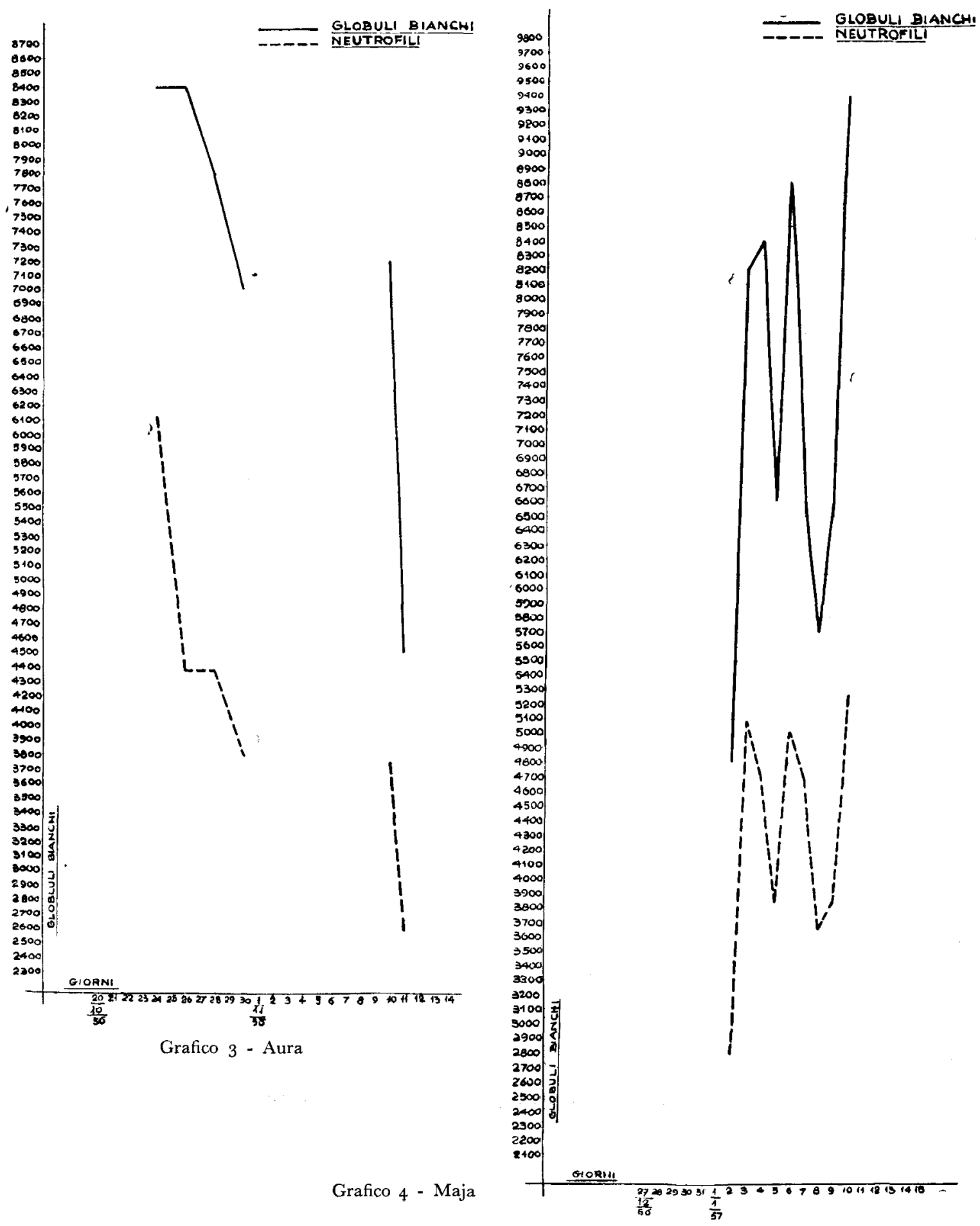


nostre personali osservazioni globuli bianchi 7.000 con $31 \%$ di granulociti neutrofili. Ad un esame successivo: Gl.B. 7.800 - Gran. n. 40\%. - Data l'importanza della fenomenologia fu subito iniziata una terapia vaccinica e antibiotica e consigliata vitamina $\mathrm{B}_{1} 2$ a forti dosi. Il paziente migliorò notevolmente e l'analisi diede al controllo Gl.B. 9. Ioo con 6o\% di neutrofili (grafico I).

Messi in sospetto dall'andamento dei granulociti del candidato credemmo opportuno indagare sulla famiglia. Nulla ci fu dato sapere degli ascendenti e collaterali sia della famiglia paterna che materna del candidato (grafico 5).

Il padre, Ugo, ha avuto tutte malattie a decorso protratto. Nell'infanzia i comuni esantemi. All'età di 23 anni ematuria e pielonefrosi da calcolo incuneato per cui fu operato di nefrectomia sinistra a 24 anni. La guarigione fu assai lenta e dolorosa. A 29 anni nuovo episodio di ematuria e calcolosi renale destra curata con idroterapia. Dai 20 anni emorroidi e digestioni difficili e lente. Spesso piccoli furuncoli. All'esame obbiettivo presentava: fegato all'arco, dolicosigma,

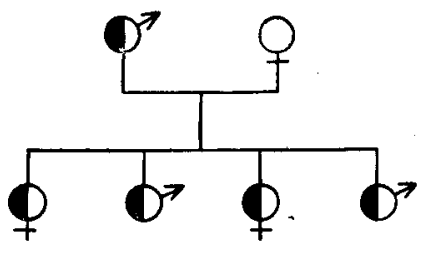

(D) granulopenia Grafico 5 emorroidi con prolasso. Gli altri dati erano tutti negativi. Un esame di sangue praticato in precedenza aveva dato Gl.B. 5.900 con $4 \mathrm{I} \%$ di neutrofili (grafico 2).

La madre, Guglielmina, è stata sempre bene e gli esami di sangue da noi praticati furono sempre normali.

Tabella I - Francesco

\begin{tabular}{|c|c|c|c|c|c|c|c|c|}
\hline Data & Gl. B. $/ \mathrm{mm}^{3}$ & Gr.N. $/ \mathrm{mm}^{3}$ & N. $\%$ & E. $\%$ & B. $\%$ & M. $\%$ & L. $\%$ & Metam. \% \\
\hline $19^{-}$I-1 $95^{6}$ & 7.800 & 3.120 & 40 & 3 & - & 5 & $5^{2}$ & - \\
\hline $8-3-1056$ & 8.300 & 3.818 & $4^{6}$ & I & - & 9 & 44 & - \\
\hline $12-5-1956$ & 9.100 & $5 \cdot 460$ & 60 & I & - & 4 & 35 & 一 \\
\hline $6-1$ I-1956 & 6.100 & 2.928 & $4^{8}$ & 2 & I & 7 & $4 \mathrm{I}$ & $\mathbf{I}$ \\
\hline $8-1$ I-1 $95^{6}$ & 8.400 & 6.216 & 74 & - & - & 3 & $2 \mathrm{I}$ & 2 \\
\hline I I-I I-1 $95^{6}$ & 7.100 & $4 \cdot 544$ & 64 & I & - & I I & 22 & 2 \\
\hline 12-1 I-1956 & 6.700 & 4.824 & 72 & I & - & 9 & 16 & 2 \\
\hline I 4-I I - I 956 & 7.200 & 5.832 & $8 \mathbf{I}$ & - & - & 4 & 15 & - \\
\hline 16-1 I-1956 & 6.700 & $4 \cdot 154$ & 62 & 2 & - & 7 & 28 & I \\
\hline I9-I 1-I 956 & 7.200 & $5 \cdot 328$ & 74 & - & - & 3 & $2 \mathbf{I}$ & 2 \\
\hline 2I-II-I 956 & 6.600 & 3.696 & $5^{6}$ & - & - & 7 & 37 & 一 \\
\hline $24-11-1956$ & 6.800 & 4.692 & 69 & I & - & 3 & 27 & - \\
\hline
\end{tabular}

Tutte le determinazioni del globuli bianchi di Francesco sono state effettuate quando già stava sotto l'azione di forti dosi di vitamina $B^{12}$. 
La sorella maggiore, Aura, di anni 22, nubile, ha avuto nell'infanzia pertosse, varicella, morbillo. A I I anni polmonite sinistra con pleurite. Più avanti tonsilliti frequenti e conseguente tonsillectomia (grafico 3).

A I5 anni iniziò un'acne che dura tuttora e spesso impetiginizza.

L'esame obbiettivo al momento in cui si presentò a noi era negativo per gli organi interni. Sulla cute del viso acne impetiginizzata diffusa e ipertricosi, limitata essa pure al viso. Un'analisi fatta precedentemente dava gl.b. 6.900 con 50\% di granulociti neutrofili.

Maja, sorella di anni I5. A.P.R.: Nell'infanzia pertosse e scarlattina. Tonsilliti frequenti. A I 3 anni appendicectomia dopo molti attacchi acuti del male. Attualmente astenica. E.O. Cute pallida con eczema all'ascella. Tonsille ipertrofiche, criptiche. Lordosi di modico grado. Apparato respiratorio indenne. Cuore lievemente a palla. Toni forti. Gli altri dati sono negativi (grafico 4).

Tab. 2 - Ugo (padre)

\begin{tabular}{|c|c|c|c|c|c|c|c|c|}
\hline Data & Gl. B. $/ \mathrm{mm}^{3}$ & G. N. $/ \mathrm{mm}^{3}$ & N. $\%$ & E. $\%$ & B. $\%$ & M. $\%$ & L. $\%$ & Metam. $\%$ \\
\hline 6-I I-1956 & $5: 400$ & 3.402 & 63 & 1 & I & 2 & 32 & I \\
\hline 8- I I-1 956 & 4.400 & 2.112 & $4^{8}$ & 4 & - & 3 & 43 & 2 \\
\hline I0-11-1956 & 3.000 & 1.710 & 57 & 2 & - & 4 & 37 & 一 \\
\hline $12-11-195^{6}$ & 5.600 & 2.688 & $4^{8}$ & - & 2 & $I$ & 49 & 一 \\
\hline I 4-I I-1956 & 3.600 & I. 728 & 48 & - & 一 & Io & $3^{8}$ & 4 \\
\hline I6-I I-I 956 & 3.200 & I. 632 & $5 I$ & 4 & 1 & 9 & 33 & 2 \\
\hline I9-1 I-I956 & 4.800 & 3.600 & 75 & 2 & 2 & 4 & I6 & $\mathbf{I}$ \\
\hline 2 I-1 I-I 956 & 2,100 & I. I 76 & $5^{6}$ & - & 一 & 12 & $3 \mathbf{I}$ & $\mathbf{I}$ \\
\hline $24^{-1}$ I-I 956 & 3.200 & I. 536 & $4^{8}$ & 2 & 2 & 4 & 44 & - \\
\hline 26 - I I-I $95^{6}$ & 2.600 & $1.35^{2}$ & $5^{2}$ & I & 4 & 4 & 37 & 2 \\
\hline $3^{30-11-1956}$ & 3.600 & I. 728 & $4^{8}$ & 4 & 2 & 12 & 34 & 一 \\
\hline $3^{-12-1956}$ & 4.200 & 2.226 & 53 & 3 & I & 9 & $3^{2}$ & 2 \\
\hline $5^{-12-195^{6}}$ & 2.700 & I. $45^{8}$ & 54 & 4 & 一 & 6 & 34 & 2 \\
\hline $7-12-195^{6}$ & 3.100 & 1. 736 & 56 & I & - & 18 & 25 & $一$ \\
\hline 10-12-1956 & 1,800 & 900 & 50 & 3 & $\mathbf{I}$ & Io & $3^{6}$ & 一 \\
\hline $12-12-195^{6}$ & 2.800 & 1.400 & 50 & 4 & 3 & 20 & 23 & 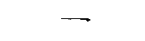 \\
\hline $14-12-195^{6}$ & 4.600 & 2.116 & 46 & 3 & 1 & 23 & 27 & 一 \\
\hline $17-12-195^{6}$ & 3.600 & 1.476 & $4^{I}$ & 一 & I & 9 & $4^{8}$ & - \\
\hline 19-12-1956 & 4.800 & $2.35^{2}$ & 49 & 3 & - & I I & 37 & 一 \\
\hline 21-12-1956 & 3.500 & 2.030 & $5^{8}$ & 一 & - & 7 & 35 & - \\
\hline 2- $1-1957$ & $5 \cdot 4^{\circ 0}$ & 2.268 & $4^{2}$ & 2 & $I$ & 5 & $4^{8}$ & 2 \\
\hline 3- I-1957 & 3.400 & $1.83^{6}$ & 54 & 3 & - & 9 & 34 & 一 \\
\hline 4- I-I 957 & 4.000 & 2.120 & 53 & $\mathbf{I}$ & I & 8 & 37 & - \\
\hline 5- I-1957 & 2.800 & I. 624 & 58 & 3 & 3 & 8 & 28 & - \\
\hline 12- 1-1957 & 3.200 & 1.248 & 39 & 3 & - & $2 \mathrm{I}$ & 37 & - \\
\hline
\end{tabular}


Torrioli Riggio G.: Considerazioni su una famiglia di granulopenici

Tab. 3 - Guglielmina (madte)

\begin{tabular}{|c|c|c|c|c|c|c|c|c|}
\hline Data & GI. B. $/ \mathrm{mm}^{3}$ & Gr. N. $/ \mathrm{mm}^{3}$ & N. $\%$ & E. $\%$ & B. $\%$ & M. $\%$ & L. $\%$ & Metam. $\%$ \\
\hline 2-I-I957 & 6.800 & $3.74 \mathrm{O}$ & 55 & 4 & - & 3 & $3^{8}$ & - \\
\hline 3-I-I 957 & 5.800 & 4. II 8 & 71 & 3 & I & 3 & 22 & - \\
\hline $4^{4-I-I 957}$ & 5.500 & $4.73^{\circ}$ & 86 & 1 & 一 & 5 & 8 & $\ldots$ \\
\hline 5-I-I 957 & 6.600 & 5.016 & 76 & I & - & 10 & I3 & - \\
\hline
\end{tabular}

Tab. 4 - Aura (sorella)

\begin{tabular}{|c|c|c|c|c|c|c|c|c|}
\hline Data & Gl. B. $/ \mathrm{mm}^{3}$ & Gr.N. $/ \mathrm{mm}^{3}$ & N. $\%$ & E. $\%$ & B. $\%$ & M. $\%$ & L. $\%$ & Metam. $\%$ \\
\hline $24-$ I I-ז $95^{6}$ & 8.400 & $6.13^{2}$ & 73 & 3 & - & 9 & I 5 & $-\cdots$ \\
\hline $27-\operatorname{II}-195^{6}$ & 8.400 & $4 \cdot 368$ & $5^{2}$ & 5 & I & 5 & 37 & - \\
\hline $28-11-195^{6}$ & 7.800 & 4.368 & $5^{6}$ & 4 & - & 一 & $4^{\circ}$ & - \\
\hline $3^{0-1}$ I-1956 & 7.000 & 3.780 & $3^{8}$ & 6 & 2 & 3 & $5 I$ & - \\
\hline Io- $1-1957$ & 7.200 & 3.744 & $5^{2}$ & 9 & - & 8 & $3^{I}$ & - \\
\hline I I- I-I 957 & 4.500 & 2.565 & 57 & 7 & - & 9 & 27 & - \\
\hline
\end{tabular}

Tab. 5 - Maja (sorella)

\begin{tabular}{|c|c|c|c|c|c|c|c|c|}
\hline Data & Gl. B. $/ \mathrm{mm}^{3}$ & Gr. $\mathrm{N} . / \mathrm{mm}^{3}$ & N. $\%$ & E. $\%$ & B. $\%$ & M. $\%$ & L. $\%$ & Metam. $\%$ \\
\hline 2-1-1957 & 4.800 & 2.784 & $5^{8}$ & 4 & - & 7 & $3 \mathrm{I}$ & - \\
\hline 3-1-1957 & 8.200 & 5.084 & 62 & 3 & - & 12 & 23 & 一 \\
\hline $4^{-1-1}-1957$ & 8.400 & 4.704 & $5^{6}$ & - & 一 & 6 & $3^{8}$ & - \\
\hline $5^{-1-1957}$ & 6.600 & 3.828 & $5^{8}$ & 2 & 2 & 9 & 29 & - \\
\hline 6-1-1957 & 8.800 & 5.016 & 57 & 3 & - & 9 & $3^{I}$ & - \\
\hline $7^{-1-1957}$ & 6.600 & 4.686 & 71 & I & 一 & 3 & 25 & - \\
\hline 8-1-1957 & 5.600 & 3.640 & 65 & 3 & - & 4 & 24 & 4 \\
\hline 9-I-1957 & 6.600 & 3.828 & $5^{8}$ & - & - & 15 & 27 & - \\
\hline I0-I-I 957 & $9 \cdot 400$ & 5.264 & $5^{6}$ & 2 & - & 15 & 27 & - \\
\hline
\end{tabular}

Novello, fratello di anni 7. Nella prima infanzia IV malattia. Operato di ernia scrotale destra a 5 anni. Qualche tonsillite. Nell'inverno bronchiti frequenti.E.O. Faringite eritematosa, tonsille ipertrofiche, anfrattuose. Numerosi denti cariati. Torace e addome negativi. Ad un esame praticato precedentemente gl.b. 9.500. Dopo la bronchite gl.b. i r.60o - gran. neutr. $58 \%$.

Iniziammo subito un esame sistematico del numero dei globuli bianchi e della formula leucocitaria in tutta la famiglia e ne riportiamo $i$ dati in grafici e tabelle. 
Tab. 6 - Novello (fratello)

\begin{tabular}{|c|c|c|c|c|c|c|c|c|}
\hline Data & Gl.B. $/ \mathrm{mm}^{3}$ & Gr. N. $/ \mathrm{mm}^{3}$ & N. $\%$ & E. $\%$ & B. $\%$ & M. $\%$ & L. $\%$ & Metam. $\%$ \\
\hline 2-1-1957 & $9 \cdot 400$ & 7.050 & 75 & 4 & $\cdots$ & 5 & 16 & - \\
\hline $3^{-1-1} 957$ & 8.800 & 6.600 & 75 & 6 & I & 8 & 10 & - \\
\hline 9-10-1957 & $9 \cdot 400$ & 6.768 & $7^{2}$ & I & - & 6 & $2 \mathrm{I}$ & - \\
\hline 10-10-1 957 & 6.600 & 4.884 & 74 & I & I & 3 & $2 \mathrm{I}$ & - \\
\hline I I-10-1957 & 5.800 & $3 \cdot 306$ & 57 & - & - & 9 & 34 & - \\
\hline 12-10-1957 & 5.000 & 2.400 & $4^{8}$ & I & - & Io & $4^{I}$ & - \\
\hline 13-10-1957 & $5 \cdot 100$ & $2.95^{8}$ & $5^{8}$ & I & 一 & I & $4^{I}$ & - \\
\hline 14-10-1957 & 7.900 & 4.819 & 65 & I & - & 5 & 33 & - \\
\hline 15-10-1957 & 9.000 & 6.210 & 69 & I & - & 4 & 26 & - \\
\hline
\end{tabular}

Per la madre, insegnante, e per il bambino, data la negatività dei primi reperti e le occupazioni o l'età degli interessati non abbiamo potuto insistere ${ }^{1}$.

Le analisi vennero sempre praticate la mattina a digiuno, da noi stessi e con le solite tecniche di conteggio e di colorazione.

Concludendo si tratta di una granulopenia ciclica familiare e analogamente a quanto segnalato dalla grande maggioranza degli AA., va considerata come granulopenia in senso stretto, in quanto alla fase granulopenica si accompagna spesso una salita contemporanea dei linfociti che attenua o maschera addirittura il fenomeno se ci si limita alla cifra bruta dei globuli bianchi. Ricordiamo che nei casi di Cesar la reazione linfatica era tale da provocare un ingrossamento delle ghiandole linfatiche.

Si tratta nel nostro caso di una forma assai lieve: tanto da rimanere in fase subclinica nel padre, dove solo la furuncolosi o la tendenza al prolungarsi delle malattie mettono sull'avviso; da essere clinicamente muta nelle due sorelle e chiaramente manifestatasi in Francesco, nel quale la fistola continuamente recidivante e la furuncolosi insistente e a decorso anomalo hanno portato finalmente alla diagnosi. Nulla si ritrova nella madre e in un fratello minore del candidato. Tanto più importante, dal punto di vista clinico, sono questi casi limite in cui l'anomalia non dà segni conclamati di sè sì da essere direttamente individuata e va scovata come «sottofondo » patologico che solo può spiegare l'andamento ciclico di alcune affezioni infettive.

$\mathrm{Ci}$ sembra assolutamente indiscutibile la familiarità lereditaria,evidentemente legata al padre, anche se disgraziatamente ci è mancata l'osservazione diretta dei familiari di questo e se le notizie anamnestiche dirette sono praticamente mute. Da

1. In corso di stampa ci è stato condotto il piccolo Novello: Egli infatti, in un periodo di 2 mesi ha sofferto successivamente di influenza, tipo AI Singapore, con complicanze broncopolmonari gravi; pertosse e bronchite. Abbiamo trovato un esame obbiettivo praticamente negativo; l'esame quotidiano e prolungato dei glcbuli bianchi, invece, ci ha permesso di dimostrare"anche in lui un andamento ondulante, con cicli, questa volta, settenari, di cui diamo un esempio nella tabella 6. 
notare come in questa famiglia si tratti di cicli brevi (4-5 giorni), che si iscrivono forse in cicli più lunghi bi o trisettimanali. Da notare inoltre la nessuna influenza del sesso, mentre nella casistica di Reimann e de Berardinis predominavano i maschi ( I o su i6). Nessuna influenza dell'età da I 5 a 47 anni. È chiaro infatti che i casi sicuramente familiari non possono presentare nè età di inizio, nè età di scomparsa dell'anomalia che dura tutta la vita. Quando si sente parlare di inizio e di fine si deve pensare esclusivamente alle manifestazioni cliniche secondarie che possono presentare carattere episodico. Tutto questo vale solo per le forme documentatamente ereditarie; d'accordo con Wintrobe non escludiamo affatto la possibilità di granulopenie croniche a decorso protratto e ad andamento ciclico, totalmente estranee alla forma di cui ci stiamo occupando e che possono presentare remissioni complete. Son questi i casi in cui anche l'ipotesi di Sturgis di un'attività ciclicamente aggressiva di un eventuale virus, può essere ripresa in considerazione. Nelle forme sicuramente familiari invece è ovvio che le manifestazioni infettive debbano essere considerate come secondarie sia per il loro stabilirsi, sia per il loro andamento prolungato e ciclico.

Pur partendo quindi da una base ereditaria resta completamente aperta la discussione sul come la anomalia genica si manifesti nel fenotipo.

Come abbiamo visto nella impostazione, le ipotesi fondamentali si riducono a due: o una variazione primitiva ciclica dell'attività degli organi emopoietici o una variazione dello stato funzionale degli organi e sistemi deputati alla regolazione funzionale degli organi emopoietici stessi. Già ad un superficiale esame è evidente come la prima ipotesi lasci completamente insoddisfatti. Il midollo osseo ed i tessuti reticolare e linfatico, come e forse più di ogni altro organo sono sotto il controllo di un gran numero di altri organi ed apparati (dal sangue circolante al sistema nervoso) che ne regolano così strettamente la produzione da renderla rispondente ad ogni minima variazione dei bisogni dell'organismo. Parlar quindi di variazioni dell'attività degli organi emopoietici equivale a parlare di oscillazioni del complessissimo sistema della loro regolazione funzionale.

Per quel che riguarda una regolazione puramente ormonica primitivamente affetta da ciclizzazione, quasi tutti gli AA. son concordi nel dire che se è facile postularla altrettanto difficile e praticamente fino ad oggi impossibile è darne una soddisfacente documentazione. Del sesso e dell'età abbiamo detto; anche la famiglia da noi studiata tende ad escludere ogni importanza in proposito. In particolar modo per il sesso femminile, l'attenzione di tutti gli studiosi è stata portata particolarmente sulla possibile importanza determinante del ciclo mestruale. In un caso di Muratova, infatti, esisteva un certo nesso tra ciclo mestruale e neutropenia; nel nostro caso non è risultato dimostrabile tale nesso e in una paziente di Reimann e de Berardinis la condizione persistette durante una gravidanza.

L'altro organo che ha richiamato e richiama l'attenzione in argomento è la milza. Come è noto dalla leucopenia bantiana, dai dati sperimentali di Torrioli e coll., dalla concezione clinica di Franck e di Ferrata di inibizione splenomidollare, dai reperti clinici infine, di Wiseman e Doan, la milza svolge un'azione frenatrice su molte funzioni del midollo osseo tra cui la granulopoiesi. E ciò anche nel normale. Una 
esaltazione patologica di detta funzione, nel corso del cosidetto ipersplenismo, può portare a cifre bassissime di neutrofili circolanti. Da notare come suggestivo il fatto che mentre l'esaltazione funzionale della milza porta alla neutropenia, provoca d'altro canto una salita dei monociti e dei linfociti in perfetta armonia con quanto si è visto nella neutropenia ciclica familiare.

Ricordiamo anche come, sempre in campo splenico, Möschlin trovando in un caso una notevole eosinofilia nel puntato splenico, durante le fasi neutropeniche, avanzò l'ipotesi di una forma allergica. A questo proposito ricorderemo anche che un malato di Sturgis, tecnico dentista, vide scomparire i suoi disturbi cambiando lavoro. Non si sa però se la neutropenia ciclica sia rimasta o meno.

Ogni conclusione in argomento va però innanzi tutto passata al vaglio dei risultati della splenectomia che è stata effettivamente praticata in molti di questi casi. Se nella milza fosse stata da ricercare la causa prima della ciclicità il risultato della splenectomia avrebbe dovuto consistere in un'abolizione del ciclo. Invece, mentre tutti gli AA. sono concordi nel dichiarare buoni gli effetti dell'intervento e nel ritenere quest'ultimo l'unica terapia capace di beneficare durevolmente i casi gravissimi, sono anche tutti concordi nel constatare che il ciclo si svolge ugualmente quantunque a livello più alto. Malati che nel momento della caduta raggiungevano l'agranulocitosi quasi completa, dopo l'intervento hanno diminuzioni compatibili con un discreto benessere. Ci sembra questo un esempio chiarissimo di quell'ipersplenismo relativo già perfettamente inquadrato dal nostro Ferrata. La milza pur essendo estranea come causa prima alla genesi del processo, interviene sempre come organo inibitore della funzione granulopoietica ed esaltatore della linfopoietica; la sua asportazione, quindi, migliora sintomaticamente la situazione senza modificare il momento patogenetico fondamentale, di natura ereditaria, agente attraverso un meccanismo tuttora ignorato. Oggi infatti sia i dati esistenti in letteratura, sia quelli della nostra osservazione, non consentono di andare oltre nel campo delle affermazioni sicure.

Nei nostri casi anche la risposta discreta alla somministrazione della vitamina B 12 , mentre totalmente inefficaci rimanevano dosi generose di $\mathrm{B}_{4}$, di ferro e di acido timonucleinico, non consentono per il momento di avanzare ipotesi patogenetiche.

Aggiungeremo infine che il nostro attuale indirizzo di lavoro è quello di ricercare anche nel normale una variabilità ciclica della formula leucocitaria. Se i risultati, come sembra, dovessero essere probativi la neutropenia ciclica familiare assumerebbe la veste di una patologica esaltazione di un fenomeno normale e rientrerebbe in un quadro molto più vasto che in vari campi della fisiologia e della patologia si va abbozzando e che promette risultati di estremo interesse. 


\section{Riassunto}

L'A., dopo una rapida scorsa alla relativamente scarsa letteratura sull'argomento, porta l'osservazione personale di una famiglia con granulopenia ciclica familiare e ne conferma l'ereditarietà.

\section{Bibliografia}

I. Löffler, W, e C. MaIer: Ueber einen Fall von Feltyschem Syndrom mit cyclischer Agranulocytose. Cardiologica, 12 ; 195 (I947).

2. Müller, B. et Meyrieux, A: Reunion International d'Hematologie, Paris, 24, 5, 48.

3. Imerslund, O.: A case of cyclic agranulocytosis. Acta paediatr. (Stockh) 30; 232, (1942)

4. VAHLQuist, B.: Cyclical agranulocytosis; Reports of a case with a short of the disease, Acta med. scand. (Stockh) Suppl. 170; 531 (1946).

5. Reimann and De Berardinis: Periodic (Cyclic) Neutropenia, An Entity. Blood, 4; i 109 i949.

6. Cyrus C. Sturgis: Hematology - second edition - Springfield Illinois U.S.A.

7. Cesar A. B.: Familial Chronic Malignant Neutropenia, Bol. Soc. Cubana. Pediatria. $15 ; 900,1943$.

8. S. Möschlin: La Puncion del Bazo. Ediciones Morata. Pagg. 171 - 173.

9. Wintrobe M.: Clinical Hematology. Third Edition. Chapter 19, pagg. 965-976.

10. Muratova R.: Cyklicky prubeh agramilocytosy cyklic. Cas. lék. cesk. 1947, 86 51, 1546. Ref. Excerpa med., 2; I020 (1948). 
L'A., après avoir en bref résumé la bibliographie sur le sujet - qui est, d'ailleurs, très peu nombreuse, - décrit une nouvelle famille avec granulopenie ciclique familiale et confirme le caractère héréditaire de la maladie.
The A., after a short survey of the scarce bibliography on the subject, describes a personal case of a cyclic familiar granulopenia and confirms the hereditary character of the disease.

\section{ZUSAMMENFASSUNG}

Nach kurzer Übersicht der verhältnismässig wenigen Literaturangaben berichtet Verf. über die persönliche Beobachtung einer Familie mit zyklischer Familiengranulopenie. Verf. bestätigt bilitazione di Montréal su 250 casi di paralisi 